\title{
CREENCIAS AMBIENTALES E IDEOLOGÍA EN POBLACIÓN CHILENA
}

\author{
Environmental beliefs and ideology in Chilean population
}

\author{
Emilio Moyano Díaz* \\ Gonzalo Palomo Vélez** \\ Paz Moyano Costa***
}

\begin{abstract}
RESUMEN
La crisis ambiental global presenta una pluralidad de manifestaciones sociales que en Chile, país con problemas de energía, van desde el rechazo ciudadano a proyectos de construcción de represas, hasta el retiro de iniciativas de ley relativas a la privatización de semillas en el parlamento. Se desconoce sin embargo cuáles son las creencias ambientales de los ciudadanos y la relación de estas con su ideología política y religiosa, y su eventual comportamiento pro-ambiental. Se analiza aquí las creencias ecocéntricas y antropocéntricas, y el comportamiento ambiental en chilenos adultos. Se observa que quienes se adscriben a posiciones políticas de derecha así como quienes declaran tener religión tienden a ser más antropocéntricos que ecocéntricos. Independientemente de su ideología, todos los participantes son desfavorables a la construcción de centrales nucleares y también son escépticos respecto de que cualquier gobierno que asuma pueda realizar más de lo hecho hasta ahora por proteger el ambiente.
\end{abstract}

Palabras clave: Creencias ambientales, ideologías, comportamiento pro-ambiental.

\footnotetext{
* Académico de la Facultad de Psicología, Universidad de Talca. Talca, Chile. Correo electrónico: emoyano@utalca.cl

** Facultad de Psicología, Universidad de Talca. Talca, Chile. Correo electrónico:gpalomovelez@ gmail.com

*** Universidad de Playa Ancha. Valparaíso, Chile. Correo electrónico:pelmoyano@hotmail.com
}

Artículo recibido el 12 de agosto de 2014. Aceptado el 2 de diciembre de 2014. 


\begin{abstract}
The global environmental crisis comprises a plurality of social expressions that in Chile, country with energy problems, go from the citizenship rejection to dam construction projects, to the withdrawal of draft law projects concerning the privatization of seeds in parliament. However, it is unknown what are the citizen's environmental beliefs and it's relation with their political and religious ideology, and their eventual pro-environmental behavior. Egocentric and anthropocentric beliefs and environmental behavior are analyzed here in Chilean adults. It is observed that those who ascribe to right political positions as well as those who declare to believe in a religion tend to be more anthropocentric than egocentric. Regardless of their ideology, all participants are unfavorable to the construction of nuclear power plants and are also skeptical that any government could do more than what it has been done so far to protect the environment.

Keywords: Environmental beliefs, ideologies, pro-environmental behavior.
\end{abstract}

\title{
INTRODUCCIÓN
}

\section{Politica y ambientalismo en Chile}

Muy recientemente sectores de la opinión pública en Chile levantaron voces críticas respecto de un gran proyecto de explotación minera de oro (Pascua Lama) en el norte del país -cuya extensión traspasaría la frontera cubriendo territorio argentino- por parte de empresas canadienses. Asimismo, otra iniciativa esta vez en el campo legislativo, relativa a regular la apropiación legal de nuevas semillas por parte de sus creadores y su explotación exclusiva (multinacional de alimentos Monsanto), ha sido detenida en su trámite en el parlamento chileno. Así, desde la base hasta la superestructura política de la sociedad, los ciudadanos parecen tener actitudes negativas hacia proyectos atentatorios contra el medioambiente. Y con razón. Pascua Lama significaba explotación de territorios ancestrales de la etnia diaguita lo que también incluía tocar glaciales (que lamentablemente fueron afectados por la extendida fase de 'exploración' previa), en un contexto nacional en el que se está buscando recuperar el agua como bien público y en el que a nivel internacional abundan -y se profundizan- conflictos y guerras por la energíapetróleo, pronosticándose que en el futuro lo serán por el agua ("agua sí, oro no”).

\footnotetext{
${ }^{1}$ Interesados en la lucha de la comunidad diaguita y de Huasco ver "El dorado: La sed del oro" (Mirages d' un El dorado)/Pascua Lama-Chile (documental Youtube). El atraso de las leyes ambientales en Chile permitió a Greenpeace fundar un nuevo país en un territorio no reconocido por Chile "República Glaciar nace porque en Chile existe un vacío en la legislación que no reconoce estas enormes masas de hielo como parte de su soberanía. Ni en la Constitución, ni en el Código de Aguas existe mención a los glaciares como bien público que debe protegerse activamente”. M. Asún, Director de Greenpeace
} 
Detener la iniciativa de legislar para dar cobertura legal a la apropiación de patrimonios de semilla y al cultivo de transgénicos en territorio nacional a la multinacional Monsanto aparece como una medida necesaria en un país que se precie de culto y soberano. El gobierno ha impedido la realización del proyecto de represa HidroAysén en la Patagonia chilena por no respetar adecuadamente la normativa ambiental existente.

Por otra parte, los medios de información han difundido algunas de las muchas pugnas de los ambientalistas y sus organizaciones así como del público común, cuando se refieren al ambiente y, especialmente, cuando aquellas significan "alteración del orden público". Así, se ha observado repetidamente toma de caminos, obstaculizando el transporte en Freirina contra malos olores (Emol, 2012); en Aysén y Corral por la pesca de arrastre depredatoria, lugar este último donde para denunciarlo, se reunieron los representantes de más de 50.000 pescadores de todo el país denunciándola (Diario El Ranco, 2012). Ha habido manifestaciones violentas en distintos lugares de Chile -Santiago, Valparaíso, Concepción y Coyhaiquecontra el proyecto HidroAysén (Emol, 2011). También en la Región de Valparaíso (Con-Cón) por la gravísima contaminación del aire de la refinería de petróleo homónima (BioBío Chile, 2012) y en Puchuncaví (aledaño a Con- Cón), por la contaminación del mar y sus impactos sobre la pesca y la salud de la población. Ello culminó con la emblemática muerte de un trabajador por tener exceso de mercurio en su sangre a causa de los contaminantes del complejo industrial (El Mostrador, 2011), entre otras muchas manifestaciones pro defensa del ambiente y de la calidad de vida de los habitantes, amenazada por la contaminación ambiental.

Durante las últimas décadas la discusión sobre el deterioro medioambiental y la protección de los ecosistemas ha cobrado gran importancia, configurándose como un problema de contingencia internacional (Gosovic, 1992; Mercado y Córdova, 2013). En el contexto latinoamericano, el ambientalismo o la preservación del medioambiente es aprobado por algunos sectores y considerado ambicioso por otros, quienes argumentan que se trataría de un lujo para países en vías de desarrollo y por ahora solo pertinente para países ricos (Gudynas, 1992). La región se ha visto inmersa en un sistema de tensiones internacionales, en el cual países desarrollados abogan y presionan por la protección y preservación del medioambiente de algunas regiones latinoamericanas, argumentando que serían de las pocas en el globo que aún conservan ecosistemas relativamente ajenos a la intervención humana pero, seguramente también, porque constituyen grandes depósitos de recursos naturales potencialmente apropiables. Mientras, otros actores internos verían estas intenciones conservacionistas como obstáculos al desarrollo y crecimiento económico, al ser

en Chile. Disponible en: http://www.greenpeace.org/chile/es/blogs/blog/Gracias-a-un-vacio-legalGreenpeace-funda-un-nuevo-pais-en-un-territorio-no-reconocido-por-Chile/ 
la explotación primaria de recursos naturales uno de los principales pilares de las economías latinoamericanas (Estenssoro, 2010).

El tema del ambientalismo se instaló en la agenda pública en Chile hacia principios del siglo XX, generando debates políticos sobre cómo habría de enfrentarse y cómo se mitigaría el deterioro medioambiental. En los años 30 las tesis desarrollistas sostenían que la protección de recursos naturales eran la base para el progreso del país y posteriormente, estas fueron relativizándose durante el periodo de dictadura (1973-1989) donde, sin embargo, y en línea con el nacionalismo de la época, se discutía la protección de especies y ecosistemas chilenos (Ulianova y Estenssoro, 2012). En este periodo dictatorial el discurso pro-medioambiente fue impulsado fuertemente por ONGs, organizaciones de disidencia política que acogieron y fueron bien recibidas por los nuevos movimientos sociales de las décadas del 70 y 80 relativos al ambientalismo, el pacifismo, el feminismo, entre otros (Ulianova y Estenssoro, 2012; Wahl, 1997). Posteriormente, con la recuperación de la democracia, los gobiernos de la Concertación (centro-izquierda) abogaron por un modelo que apuntaba al desarrollo sustentable, generándose algunos hitos en la institucionalización de la temática medioambiental, entre ellos, la creación en 1990 de la Comisión Nacional del Medioambiente (CONAMA) y la promulgación en 1994 de la Ley No 19.300 sobre Bases Generales del Medio Ambiente (Pizarro y Vasconi, 2004) y, más recientemente la creación del Ministerio del Medioambiente en 2010 y la sustitución de la CONAMA. Sin embargo, este avance hacia la institucionalización de temas ambientales es simultáneo -y contrasta- con el favorecimiento de políticas de extracción de recursos naturales (principalmente para exportación), con consecuencias ambientales tales como la deforestación o contaminación de las aguas (Gudynas, 2009).

Actualmente en Chile el debate medioambiental está mediado por las percepciones hacia el propio modelo de desarrollo imperante $-\mathrm{y}$ por tanto- por la adscripción a unos u otros principios políticos. Así, desde sectores de la derecha política y económica del país, comúnmente vinculados al favorecimiento del modelo económico neoliberal, se plantea que la correcta defensa del medioambiente ha de ir en línea con los fundamentos que estructuran y sustentan el libre funcionamiento del mercado y los principios de la propiedad privada, en contraste con los sectores de izquierda donde se plantea la necesidad de acabar con el modelo neoliberal y potenciar la intervención del Estado en la actividad económicaproductiva a fin de proteger el medioambiente natural (Estenssoro, 2007). Así, con distintas perspectivas o posiciones -respectivamente más antropocéntricas o más ecocéntricas- el tema del medioambiente está presente en el discurso de ambas orientaciones políticas. 


\section{CREENCIAS AMBIENTALES Y SU MEDICIÓN}

Las creencias acerca del medioambiente y enfatizan una visión globalmente ecocéntrica al respecto es una meta deseable en la educación de los jóvenes de hoy en el mundo. Muchos de estos llegan a la educación superior con creencias y comportamientos ecocéntricos y en algunos estudios recientes se ha buscado determinar si estos son mantenidos, reforzados o debilitados durante y según su formación universitaria (Heyl, Moyano y Cifuentes, 2014).

Dada la importancia del tema ambiental y los esfuerzos de protección del medio ambiente en el mundo, las investigaciones sobre las actitudes pro-ambientales y el comportamiento ecológico se han multiplicado durante los últimos años (Amérigo, 2006). El enfoque sobre la relación hombre-naturaleza propuesto por Dunlap y Van Liere (1978) es uno de los de uso más extendido. En este, y a diferencia del paradigma social antropocéntrico dominante (el hombre como superior a los organismos naturales) (Pirages y Ehrlich, 1974), se concibió al ser humano y su relación con el mundo natural ecocéntricamente, es decir, donde el sistema natural y sus normas y restricciones eran igualmente válidas para el hombre como para todos los organismos naturales (Dunlap, 2008; Dunlap y Van Liere, 1984). Así, se entendía al hombre como un animal más en el mundo, sin posición preferente ni de superioridad para la explotación del ambiente. Esto convergía con una concepción de la tierra como un organismo vivo al que hay que cuidar, y con el cual mantener una relación armónica.

Dunlap y Van Liere (1978) desarrollaron un instrumento para medir ecocentrismo y antropocentrismo denominado Nuevo Paradigma Ambiental (NEP por sus siglas en inglés), cuya versión revisada -escala Nuevo Paradigma Ecológico o NEP-R- de Dunlap, Van Liere, Merting y Jones (2000) es ampliamente utilizado en América, Europa y más recientemente en África (Ogunbode, 2013). A mayor puntaje obtenido en este instrumento mayor predominio de una perspectiva ecocentrista o compromiso con la preservación y protección del ambiente natural, en tanto bajas puntuaciones supondrían predominio de una visión antropocentrista o pro-explotación de la naturaleza (Dunlap et al., 2000; Hawcroft y Milfont, 2010).

\section{CREENCIAS AMBIENTALES E IDEOLOGÍA}

Las creencias que las personas desarrollan respecto del ambiente natural pueden verse influidas por sus tendencias ideológicas (McCright y Dunlap, 2012). Estudios realizados en USA muestran consistentemente que las personas que adscriben a orientaciones ideológicas más bien demócratas tienden a evaluar los efectos de ciertos problemas medioambientales como más dañinos y contingentes que quienes adscriben ideologías más de derecha o conservadoras (republicanos) 
(Dunlap y McCright, 2008; Dunlap, Xiao y McCright, 2001; McCright y Dunlap, 2011). Dado los diferentes efectos que ha mostrado la literatura al respecto, se ha introducido la distinción entre adherencia a un partido político y la adscripción a una ideología política. Existiría evidencia sustancial en la literatura que las personas que adscriben a un pensamiento de tipo liberal evidenciarían más actitudes a favor o pro-medioambiente en comparación a quienes se identifican con un pensamiento más conservador (Van Liere y Dunlap, 1980).

Neumayer (2004) reportó que tanto las personas que adhieren a partidos políticos de izquierda, como también quienes se consideran a sí mismos como de ideología izquierdista, tienen más probabilidad de argüir a favor de posiciones proambientales que sus contrapartes de derecha. Midiendo no la adscripción política, sino el pensamiento económico liberal, ha sido observado que este correlaciona con pensamiento de tipo anti-antropocentrista, es decir, es posible que se esté frente a una nueva generación de ciudadanos que no necesariamente entiende el liberalismo económico como la favorabilidad hacia la explotación desmedida de los recursos naturales, sino que más bien parecen superar la dicotomía entre conservación y explotación del medioambiente (Moyano-Díaz, Cornejo y Gallardo, 2011) entendiendo, tal vez, que no es sustentable el crecimiento ni el desarrollo sin equilibrio con el ambiente.

Jacques, Dunlap y Freeman (2008) analizaron la relación entre Centros de Investigación Conservadores (CTT), típicamente asociados a la defensa de los derechos de la propiedad privada, la libre empresa y la defensa nacional entre otros, y el escepticismo ante la problemática medioambiental. Mediante la identificación y selección de 141 libros (de habla inglesa) que expresaban escepticismo medioambiental -restando importancia o incluso negando problemas tales como el cambio climático, la extinción de especies de plantas y animales, contaminación atmosférica o acuática, etc. - analizaron si acaso estos se vinculaban con CTT, considerándose por vínculo la afiliación del autor a un CTT o si el libro mismo era publicado por una editorial perteneciente a un CTT. Así fue encontrado que $130(92.2 \%)$ de los 141 libros que sostenían una mirada escéptica ante el problema medioambiental estaban asociados a un CTT, principalmente por medio de la afiliación del autor a uno de estos centros.

Otra variable ideológica que ha sido relacionada con el ambientalismo es la religión o la religiosidad (Guth, Green, Kellstedt y Smidt, 1995; Raudsepp, 2001; Schultz, Zelezny, y Dalrymple, 2000; Stanford y Brewer, 2011). Una de las tesis más controversiales acerca de esta relación fue propuesta por White (1967), quien sostuvo que el ethos o la creencia central del cristianismo medieval argumentado en la Biblia, autoriza y justifica al hombre a dominar la naturaleza y sus recursos para su propia conveniencia, permitiendo así la emergencia de actitudes a favor de la explotación del medioambiente en las culturas occidentales. Si bien esta tesis 
estimuló fuertemente la investigación, la relación entre ambientalismo y religiosidad se observa poco consistente en la literatura, encontrándose incluso algunos estudios que indicarían una relación positiva entre ambas variables (Stanford y Brewer, 2011). Guth et al. (1995) reportó que el conjunto de creencias conservadoras - propias de las religiones judeo-cristianas-, la tradición religiosa y el compromiso con la misma, tienen influencia en las visiones medioambientales, con una influencia mucho más fuerte para el clero y los creyentes activos. Asimismo, Raudsepp (2001) mostró que la religiosidad subjetiva -entre otras variables- era un predictor significativo tanto para la preocupación por el ambiente como también de la conducta pro-ambiental. Una evaluación de la relación entre creencias ambientales (medidas a través del NEP-R entre otros instrumentos) y la literalidad de interpretación de la Biblia judeo-cristiana en distintos países (algunos de ellos latinoamericanos, Argentina, Colombia, Costa Rica, República Dominicana, Ecuador, El Salvador, México, Panamá, Paraguay, Perú y Venezuela) mostró que el nivel de literalidad con que se interprete la Biblia influye en las actitudes medioambientales las personas, siendo los más literales quienes evidencian mayor tendencia al antropocentrismo.

Tal como las variables ideológicas, algunas variables sociodemográficas han recibido atención en la investigación de las creencias medioambientales. En cuanto a esto, el sexo y la edad aparecen recurrentemente en la literatura sobre el tema. Sobre la primera, existen estudios que reportan que las mujeres tenderían a presentar más creencias pro-ambientales y más preocupación por los riesgos medioambientales que los hombres (Zelezny, Chua y Aldrich, 2000), sin embargo, otros estudios indicarían lo contrario (Arcury, Scollay y Johnson, 1987; Arcury, 1990; MoyanoDíaz y Palomo-Vélez, 2014), por lo que la dirección de este efecto aún no es concluyente. Respecto de la edad, ha sido planteado que la población joven -en comparación con la de edad más avanzada- pareciera presentar más creencias proambientales (Chen et al., 2011; Fransen y Meyer, 2010; Moyano-Díaz y Palomo Vélez, 2014). Estos últimos han encontrado que la población de mediana edad (29-40 años) presenta más creencias pro-ambientales en comparación con otros grupos etarios (menores y mayores a dicho rango), resultado congruente con la idea de que los jóvenes y los ancianos se mantendrían al margen de temas públicos (tales como la preocupación por el medioambiente), mientras que la participación en dichos temas alcanzaría su máximo hacia la mediana edad (Franzen y Meyer, 2010).

Así, es de interés aquí identificar las creencias ambientales -antropo o ecocéntricas- y el liberalismo económico en la población chilena joven y adulta de ambos sexos. 


\section{MÉTODO}

La presente investigación es de tipo descriptivo y correlacional y en ella participan hombres y mujeres, principalmente habitantes de la Región del Maule.

Participantes. Una muestra no probabilística de 760 personas voluntarias, cuyas edades fluctúan entre 17 y 74 años, 92.6\% de las cuales habitan la Región del Maule (Chile), mientras que el $7.4 \%$ restante proviene de ciudades comprendidas entre la Región Metropolitana y Octava Región del Bío Bío (Chillán, Santiago, Rancagua, entre otras). Un 54.7\% ( $\mathrm{n}=416)$ corresponde a mujeres, y los restantes hombres.

Instrumentos. Un cuestionario en que se incluye dos instrumentos que corresponden a: i) Una hoja en que se solicita información sociodemográfica e ideológica: sexo, edad, orientación política segmentada en 5 opciones posibles derecha, centro derecha, sin ideología, centro izquierda, izquierda- y credo religioso al que adscribe- católico, evangélico, ateo o agnóstico u otro-. También fue solicitada la opinión acerca de la construcción de centrales nucleares en Chile -a favor o en contra-, la posición o pensamiento respecto al ambiente (ecológico, neutro o proexplotación), y se preguntó por la orientación política del gobierno que cree que hará más en cuanto a protección ambiental. ii) La escala NEP-R (Dunlap et al., 2000) en su versión reducida y adaptada a población chilena por Moyano y Palomo (en revisión) está conformada por 11 ítems con formato de respuesta Likert de cinco puntos ( 1 =fuertemente en desacuerdo, $2=$ en desacuerdo, $3=$ ni de acuerdo ni en desacuerdo, $4=$ de acuerdo, 5= fuertemente de acuerdo) dando cuenta de dos grandes dimensiones; ecocentrismo y antropocentrismo. La escala total cuenta con una confiabilidad Alfa de Cronbach de 0.757 . iii) La Escala de Conducta Ecológica (ECE) de Weigel y Weigel (1978), en su versión adaptada por Hernández, Suárez, MartínezTorvisco y Hess (1997) de 8 ítems, con formato respuesta Likert de cinco puntos ( 1 = nunca, 2 = casi nunca, $3=$ ocasionalmente, $4=$ a menudo, $5=$ muy a menudo) que recoge la frecuencia de realización de conductas ecológicas, por ejemplo, compra de productos no contaminantes, promoción del cuidado del medioambiente y reciclaje, entre otras. iv) La escala de Liberalismo Económico (ELE) de Kilbourne, Beckmann y Thelen (2002) de 3 ítems con formato de respuesta Likert de siete puntos ( 1 =fuertemente en desacuerdo a 7 =fuertemente de acuerdo) que evalúa la presencia y grado de pensamiento económico de las personas.

Procedimiento. Los participantes fueron encuestados en los diferentes barrios de las ciudades concernidas siguiendo una lógica de disponibilidad, es decir, de quienes aceptaba participar al ser requeridos al entrar o salir de supermercados o almacenes de barrios, portales de sus casas, o en sus casas directamente. Antecediendo la entrega del cuestionario se hacía leer al posible participante un consentimiento informado dando a conocer las garantías de confidencialidad y anonimato del estudio. Para el análisis de resultados se utilizó SPSS 17 y estadística no paramétrica. 


\section{RESULTADOS}

Ideologías. El mayor porcentaje de los encuestados adhiere a la ideología política de izquierda y centro izquierda $(41,1 \%)$, seguidos por quienes no adhieren a ninguna ideología en particular $(33,7 \%)$ y los que adscriben a una ideología de derecha o centro derecha $(25,3 \%)$. Asimismo, un $80.9 \%$ de los encuestados declara tener creencias religiosas y un $19.1 \%$ afirma ser ateo o agnóstico. Adscripción política y religiosa están relacionadas de modo que quienes adhieren a ideología de derecha tienden a presentar creencias religiosas, en tanto que quienes se identifican más con ideología de izquierda no lo hacen $\left(x^{2}=57.975, p<0.01\right)$. No se observa asociación entre adscripción política y diferenciación hacia la construcción de centrales nucleares en Chile, ya que la gran mayoría de la población encuestada se opone a la construcción de las mismas $\left(\mathrm{x}^{2}=2.07, p=0.354\right)$.

En cuanto a las creencias religiosas se observa que tanto por sexo $\left(\mathrm{x}^{2}=7.0\right.$, $p=0.08)$ como por edad $\left(x^{2}=0.904, p=0.342\right)$ adherir a una creencia religiosa tiende a ser la norma (figuras 1 y 6 ). Asimismo, al analizar la orientación política de los participantes se observa que no importando el sexo $\left(x^{2}=21.3, p=0.000\right)$ ni la edad $\left(x^{2}=23.6, p=0.000\right)$, la ideología de derecha tienden a ser la menos frecuente (figuras 2 y 7). Por otro lado, el análisis de las expectativas de los participantes respecto de qué tipo de gobierno hará más por el medioambiente, ajustado tanto por sexo $\left(\mathrm{x}^{2}=16.8, p=0.000\right)$ como por edad $\left(\mathrm{x}^{2}=21.8, p=0.000\right)$ muestra que mayoritariamente se cree que dará lo mismo el signo político de un futuro gobierno en cuanto a su eventual preocupación por el medioambiente, a excepción de los adultos quienes creen levemente más que un gobierno de izquierda podría hacer más al respecto (figuras 3 y 8). Respecto de la construcción de centrales nucleares en Chile los participantes no importando sexo $\left(x^{2}=15.2, p=0.000\right)$ ni edad $(\mathrm{x} 2=5.19, p=0.023)$ evidencian una clara tendencia en contra de su construcción. La autopercepción de los participantes sobre su orientación ambiental reportada, por sexo $\left(\mathrm{x}^{2}=24.9, p=0.000\right)$ y edad $\left(\mathrm{x}^{2}=2.96, p=0.227\right)$ respectivamente, muestra mayores frecuencias para un pensamiento de tipo ecológico y casi nulas para la adscripción a un pensamiento pro-explotación (figuras 5 y 10).

\section{DIFERENCIAS EN ANTROPOCENTRISMO Y ECOCENTRISMO SEGÚN IDEOLOGÍA}

Una evaluación de las diferencias por orientación política y creencia religiosa en relación a creencias ambientales muestra que las personas de orientación política de derecha son más antropocéntricas que quienes adscriben a una orientación izquierdista $(\mathrm{U}=33904, p<0.01)$. Igual acontece entre quienes 
adscriben a alguna religión ya que son más antropocéntricos que quienes no tienen identificación religiosa $(\mathrm{U}=22120, p<0.01)$. Por otro lado, no hay diferencias entre eco y antropocentristas en cuanto a la construcción de centrales nucleares en el país, posiblemente debido a que la mayoría de los participantes se encuentra en desacuerdo con su eventual construcción ( $\mathrm{U}=60328$, $p=0.673$ ).

Por otra parte, respecto del comportamiento pro-ambiental o ecológico se ha observado para la muestra total altos puntajes de realización de los comportamientos; "Comprando productos con envases re-usables o reciclables" y "Sacando o limpiando basura que no era la suya" en tanto los comportamientos de más baja realización corresponden a; "Haciendo compostaje con los restos de alimentos" y "Caminando o andando en bicicleta para ahorrar bencina". Según ideología política se observa que los participantes de derecha realizan más frecuentemente "Sacando o limpiando basura que no era la suya", mientras los de izquierda realizan más "Comprando productos con envases re-usables o reciclables", más que cualquier otro comportamiento pro-ambiental (tabla 1).

Tabla 1.

Distribución de los puntajes de la ECE por ítems y escala total y en participantes de orientación política de derecha e izquierda.

\begin{tabular}{|c|c|c|c|c|c|c|}
\hline \multirow{2}{*}{ ECE } & \multicolumn{2}{|c|}{ Total $(\mathrm{n}=776)$} & \multicolumn{2}{c|}{ Derecha $(\mathrm{n}=204)$} & \multicolumn{2}{c|}{ Izquierda (n=316) } \\
\cline { 2 - 7 } & Media & DT & Media & DT & Media & DT \\
\hline $\begin{array}{c}\text { Buscando modos } \\
\text { de reutilizar cosas }\end{array}$ & 2.85 & 1.08 & 2.61 & 1.17 & 3.06 & 1.08 \\
\hline Reciclando papel & 2.51 & 1.23 & 2.65 & 1.29 & 2.53 & 1.14 \\
\hline $\begin{array}{c}\text { Reciclando latas o } \\
\text { botellas }\end{array}$ & 2.44 & 1.23 & 2.41 & 1.27 & 2.63 & 1.19 \\
\hline $\begin{array}{c}\text { Alentando a } \\
\text { amigos de la } \\
\text { familia a reciclar }\end{array}$ & 2.53 & 1.31 & 2.59 & 1.39 & 2.72 & 1.30 \\
\hline $\begin{array}{c}\text { Comprando } \\
\text { productos con } \\
\text { envases re-usables } \\
\text { o reciclables }\end{array}$ & 3.22 & 1.20 & 2.96 & 1.23 & 3.34 & 1.18 \\
\hline $\begin{array}{c}\text { Sacando o } \\
\text { limpiando basura } \\
\text { que no era la suya }\end{array}$ & 3.01 & 1.29 & 3.22 & 1.32 & 3.09 & 1.28 \\
\hline $\begin{array}{c}\text { Haciendo } \\
\text { compostaje con } \\
\text { los restos de } \\
\text { alimentos }\end{array}$ & 2.25 & 1.33 & 2.37 & 1.40 & 2.25 & 1.42 \\
\hline
\end{tabular}




\begin{tabular}{|c|c|c|c|c|c|c|}
\hline $\begin{array}{c}\text { Caminando } \\
\text { o andando en } \\
\text { bicicleta para } \\
\text { ahorrar bencina }\end{array}$ & 2.25 & 1.53 & 3.16 & 1.53 & 2.86 & 1.52 \\
\hline ECE global & 2.72 & 0.83 & 2.74 & 0.83 & 2.81 & 0.89 \\
\hline
\end{tabular}

Fuente: Elaboración propia.

La relación entre ecocentrismo-antropocentrismo y comportamiento proambiental es la esperada -positiva y significativa- aunque de baja magnitud. Así, quienes son más ecocéntricos ejecutan más comportamientos pro-ambientales (rho=0.166, $p=0.01)$ que quienes tiende a ser más antropocéntricos.

Por otro lado, en relación al liberalismo económico se observa que quienes adscriben a ideologías de centro derecha y derecha presentan mayores niveles de pensamiento económico liberal que quienes se identifican con ideologías de centro izquierda e izquierda $\left(x^{2}=38.95, p=0.000\right)$. Por el contrario, en cuanto a la relación entre religiosidad y pensamiento económico liberal, se aprecia que quienes informan no tener creencias religiosas tienden a tener menos pensamiento económico liberal, que quienes sí informan tenerlas $\left(\mathrm{x}^{2}=11.26, p=0.001\right)$.

\section{DISCUSIÓN Y CONCLUSIONES}

La discusión acerca del deterioro medioambiental en América Latina y particularmente en Chile está atravesada por orientaciones políticas (Ulianova y Estenssoro, 2012; Wahl, 1997), posturas frente al desarrollo económico (Estenssoro, 2007) y la propia contingencia nacional (muchas veces a partir de la presión de diversos grupos ambientalistas). En este trabajo se reporta las creencias ambientales -eco y antropocéntricas- de jóvenes y adultos chilenos y su relación con variables ideológicas. El análisis de la población estudiada permite constatar una mayoría de personas que adscriben a una orientación política de izquierda o centro izquierda y una marcada tendencia a la religiosidad. Esto último parece concordar con formulaciones que afirman que a pesar de que la Iglesia católica en Chile haya perdido en el último tiempo parte de su poder, ello no implicaría necesariamente una población más secular, sino que más bien estaríamos en presencia de una transformación hacia el pluralismo religioso en América Latina (Parker, 2009). Asimismo, se observó que religiosidad y adscripción política se encuentran relacionadas y que, independientemente del signo de esta última, nadie está de acuerdo con que se construyan centrales nucleares en Chile. Por otro lado, las personas mayoritariamente no tienen expectativas positivas respecto de lo que 
puedan hacer futuros gobiernos por el medioambiente, y aquello es independiente del signo político que ellas puedan tener.

En cuanto a las diferencias en las creencias ambientales según ideología política, los resultados coincidieron con lo tradicionalmente encontrado en la literatura, dando cuenta que quienes adscriben a ideologías de izquierda tienden a presentar más creencias ecocéntricas que quienes adhieren a orientaciones de derecha (Dunlap y McCright, 2008; Dunlap et al., 2001; Jacques et al., 2008; McCright y Dunlap, 2011; Neumayer, 2004; Van Liere y Dunlap, 1980). Asimismo, los resultados indican que las personas que declaran tener alguna creencia religiosa tenderían a mantener pensamientos más antropocéntricos que quienes se consideran ateos o agnósticos, concordando con la mayoría de los estudios sobre este tema (Guth et al., 1995; Raudsepp, 2001; Schultz et al., 2000; Stanford y Brewer, 2011).

De manera similar que en otros estudios (Moyano, Cornejo y Gallardo, 2011; Moyano-Díaz y Palomo-Vélez, 2014; Pulido et al., 2012; Scott y Willits, 1994; Thapa, 1999; Vozmediano y San Juan, 2005) la relación entre creencias ambientales y conductas pro-ambientales resultó ser positiva y baja, diferenciándose por adscripción política de modo tal que las conductas ecológicas más frecuentes para quienes se identifican con ideología de derecha e izquierda no son las mismas. Asimismo, la relación entre el pensamiento económico liberal y la adscripción política arrojó resultados esperados, ya que quienes adscriben a una ideología derechista tienen probablemente más pensamiento económico liberal en comparación con quienes tienen una orientación izquierdista (Estenssoro, 2007). Sin embargo, esto no significa que necesariamente quienes adscriben a una orientación de derecha mantengan pensamientos pro-explotación del medioambiente ya que se ha reportado que el pensamiento económico liberal así como las creencias proambientales pueden coexistir, evidenciando -quizás- una posible superación de la dicotomía entre conservación y explotación del medioambiente (Moyano-Díaz et al., 2011).

En suma, aquí se da cuenta del panorama ideológico de la población joven y adulta chilena, caracterizado por creencias ambientales de nivel intermedio o neutral entre lo eco y lo antropocéntrico, una tendencia política de izquierda y centro izquierda, con una marcada presencia de religiosidad, con actitud negativa hacia la construcción de centrales nucleares y con bajas expectativas respecto de que próximos gobiernos puedan marcar diferencia respecto de lo medioambiental a lo existente hasta ahora. 


\section{REFERENCIAS}

Amérigo, María. "La investigación en España sobre actitudes proambientales y comportamiento ecológico", Medio Ambiente y Comportamiento Humano 7/2 (2006): 45-71.

Arcury, Thomas, Scollay, Susan \& Johnson, Timothy. "Sex Differences in Environmental Concern and Knowledge: The Case of Acid Rain", Sex Roles 16/7-8 (1987): 463-472.

Arcury, Thomas. "Environmental attitude and environmental knowledge", Human organization 49/4 (1990): 300-304.

BiobíoChile. (2012). Contaminación del aire: Denuncian que refinería de petróleos arroja vapores y humo en Concón. Disponible en: http://www.biobiochile. cl/2012/09/28/contaminacion-del-aire-denuncian-que-refineria-de-petroleosarroja-vapores-y-humo-en-concon.shtml. [Consultado el 6 de agosto de 2014].

Chen, Xiadong, Peterson, M. Nils, Hull, Vanessa, Lu, Chuntian, Lee, Graise, Hong, Dayong, et al. "Effects of attitudinal and sociodemographic factors on pro-environmental behaviour in urban China", Environmental Conservation 38/1 (2011): 45-52.

Diario El Ranco. (2012). Pescadores artesanales de todo Chile se reunieron en Corral. Disponible en: de http://www.diarioelranco.cl/?p=70061. [Consultado el 6 de agosto de 2014].

Dunlap, Riley. "The new environmental paradigm scale: From marginality to worldwide use", The Journal of Environmental Education 40/1 (2008): 3-18.

Dunlap, Riley \& McCright, Aaron. "A Widening Gap: Republican and Democratic Views on Climate Change", Environment: Science and Policy for Sustainable Development 50/5 (2008): 26-35.

Dunlap, Riley \& Van Liere, Kent. “The New Environmental Paradigm”, Journal of Environmental Education 9 (1978): 10-19.

Dunlap, Riley \& Van Liere, Kent. "Commitment to the dominant social paradigm and concern for environmental quality", Social Science Quarterly 65/4 (1984): 1013-1028. 
Dunlap, Riley, Van Liere, Kent, Mertig, Angela \& Jones, Robert. "New trends in measuring environmental attitudes: measuring endorsement of the new ecological paradigm: A revised NEP scale", Journal of social issues 56/3 (2000): 425-442.

Dunlap, Riley, Xiao, Chenyang \& McCright, Aaron. "Politics and the environment in America: Partisan and ideological cleavages in public support for environmentalism", Environmental Politics 10 (2001): 23-48.

El mostrador. (2011). Codelco: principal responsable de la mortal contaminación en Puchuncaví. Disponible en: http://www.elmostrador.cl/pais/2011/11/18/ codelco-principal-responsable-de-la-mortal-contaminacion-en-puchuncavi/. [Consultado el 6 de agosto de 2014]

Emol.(2011).Manifestacionesmarcanjornadadeaprobación delproyectoHidroAysén. Visitado en: http://www.emol.com/noticias/nacional/2011/05/09/480625/ manifestaciones-marcan-jornada-de-aprobacion-del-proyecto-hidroaysen. html. [Consultado el 6 de agosto de 2014]

Emol. (2012). Agrosuper: Malos olores en Freirina son un proceso biológico que no tiene solución inmediata. Disponible en: http://www.emol.com/noticias/ nacional/2012/05/22/541851/agrosuper-se-defiende-ante-criticas-porplanta-abandonada-en-freirina.html. [Consultado el 6 de agosto de 2014]

Estenssoro, Fernando. "Antecedentes sobre la protección al medio ambiente en el pensamiento de libre mercado en Chile (década de los noventa del siglo XX)", Estudios Avanzados 5/8 (2007): 35-59.

Estenssoro, Fernando. "Crisis ambiental y cambio climático en la política global: un tema crecientemente complejo para América Latina”, Universum 25/2 (2010): 57-77.

Franzen, Axel \& Meyer, Reto. "Environmental attitudes in cross-national perspective: A multilevel analysis of the ISSP 1993 and 2000", European Sociological Review 26/2 (2010): 219-234.

Gosovic, Branislav. The quest for world environmental cooperation: The case of the UN Global Environment Monitoring System. London: Routledge, 1992. 
Gudynas, Eduardo. "Los múltiples verdes del ambientalismo latinoamericano", Nueva Sociedad 122 (1992): 104-115.

Gudynas, Eduardo. "Diez tesis urgentes sobre el nuevo extractivismo", Extractivismo, política y sociedad (2009): 187-225.

Guth, James, Green, John, Kellstedt, Lyman \& Smidt, Corwin. "Faith and the Environment: Religious Beliefs and Attitudes on Environmental", American Journal of Political Science 39/2 (1995): 364-382.

Hawcroft, Lucy \& Milfont, Taciano. "The use (and abuse) of the new environmental paradigm scale over the last 30 years: A meta-analysis", Journal of Environmental Psychology 30/2 (2010): 143-158.

Hernández, Bernardo, Suárez, Ernesto, Martínez-Torvisco, Juan y Hess, Stephany. "Actitudes y creencias sobre el medio ambiente en la conducta ecológica responsable", Papeles del Psicólogo: Revista del Colegio Oficial de Psicólogos 67 (1997): 48-54.

Heyl, Marianne, Moyano-Díaz, Emilio \& Cifuentes, Luis. "Environmental attitudes and behaviors of college students: A case study conducted at a chilean university", Revista Latinoamericana de Psicología, 45/3 (2014): 487-500.

Jacques, Peter, Dunlap, Riley \& Freeman, Mark. "The organisation of denial: Conservative think tanks and environmental skepticism", Environmental Politics 17/3 (2008): 349-385.

Kilbourne, William, Beckmann, Suzanne \& Thelen, Eva. "The role of the dominant social paradigm in environmental attitudes: a multinational examination", Journal of Business Research 55/3 (2002): 193-204.

McCright, Aaron \& Dunlap, Riley. "The politicization of climate change and polarization in the American public's views of global warming, 20012010", The Sociological Quarterly 52/2 (2011): 155-194.

McCright, Aaron, \& Dunlap, Riley. "Bringing ideology in: the conservative white male effect on worry about environmental problems in the USA", Journal of Risk Research 16/2 (2012): 211-226. 
Mercado, Aexis y Córdova, Karenia. "El ambiente en la dinámica del desarrollo", Tecnología y Construcción 19/2 (2013).

Moyano-Díaz, Emilio, Cornejo, Felipe y Gallardo, Ismael. "Creencias y conductas ambientales, liberalismo económico y felicidad", Acta Colombiana de Psicología 14/2 (2011): 69-77.

Moyano-Díaz, Emilio y Palomo-Vélez, Gonzalo. "Propiedades psicométricas de la Escala Nuevo Paradigma Ecológico (NEP-R) en población chilena”, Psico 45/3 (2014): 415-423.

Neumayer, Eric "The environment, left-wing political orientation and ecological economics", Ecological economics 51/3 (2004): 167-175.

Ogunbode, Charles."The NEP scale: Measuring ecological attitudes/worldviews in an African context", Environment, development and sustainability 15/6 (2013): 1477-1494.

Parker, Cristián. “¿América Latina ya no es católica? Pluralismo cultural y religioso creciente”, América Latina Hoy 41 (2009).

Pirages, Dennis \& Ehrlich, Paul. Ark II: Social response to environmental imperatives. San Francisco: W. H. Freeman, 1974.

Pizarro, Rodrigo y Vasconi, Paola. "Una nueva institucionalidad ambiental para Chile”, Serie Análisis de Políticas Públicas 26 (2004).

Pulido, Marco, Fernández, Diana, Fernández, Mónica, Muñoz, Ana, Taramona, Iñigo y Vázquez, Jesús. "Creencias y conductas ecológicas en estudiantes de dos universidades particulares de la Ciudad de México", Revista Electrónica de Psicología Iztacala 15/4 (2012).

Raudsepp, Maaris. "Some socio-demographic and socio-psychological predictors of environmentalism", TRAMES: A Journal of the Humanities \& Social Sciences 5/4 (2001): 355.

Scott, David \& Willits, Fern. "Environmental Attitudes and Behavior A Pennsylvania Survey”, Environment and Behavior 26/2 (1994): 239-260. 
Schultz, Wesley, Zelezny, Lynnette \& Dalrymple, Nancy. "A multinational perspective on the relation between Judeo-Christian religious beliefs and attitudes of environmental concern", Environment and Behavior 32/4 (2000): 576-591.

Stanford, Virgil \& Brewer, Elizabeth. "Dominion or Stewardship: Understanding the Role of Religiosity in Forming Attitudes about the Environment through Pathway Analysis", Paper presentado en el APSA 2011 Annual Meeting Paper.

Thapa, Brijesh. "Environmentalism: The relation of environmental attitudes and environmentally responsible behaviors among undergraduate students", Bulletin of Science, Technology \& Society 19/5 (1999): 426-438.

Ulianova, Olga y Estenssoro, Fernando. "El ambientalismo chileno: La emergencia y la inserción internacional”, Si Somos Americanos 12/1 (2012): 183-214.

Van Liere, Kent \& Dunlap, Riley. "The social bases of environmental concern: A review of hypotheses, explanations and empirical evidence", Public opinion quarterly 44/2 (1980): 181-197.

Vozmediano, Laura y San Juan, César. "Escala Nuevo Paradigma Ecológico: Propiedades psicométricas con una muestra española obtenida a través de Internet", Medio Ambiente y Comportamiento Humano 6/1 (2005): 37-49.

Wahl, Peter. "Tendencias globales y sociedad civil internacional ¿̨una organización de la política mundial?", Nueva Sociedad 149 (1997): 42-50.

Weigel, Russell \& Weigel, Joan. "Environmental Concern. The development of a measure", Environment and Behavior 10 (1978): 3-15.

White, Lynn. "The Historical Roots of Our Ecologic Crisis", Science 155/3767 (1967): 1203-1207.

Zelezny, Lynnette, Chua, Poh-Pheng \& Aldrich, Christina. "New ways of thinking about environmentalism: Elaborating on gender differences in environmentalism", Journal of Social Issues 56/3 (2000): 443-457. 


\section{ANEXOS}

Figuras 1 y 6 . Frecuencia de participantes según creencias religiosas por sexo y edad
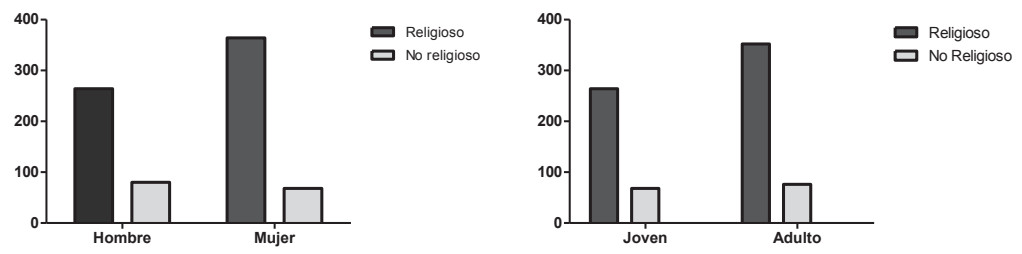

Figuras 2 y 7 . Frecuencias de participantes según ideología política por sexo y edad
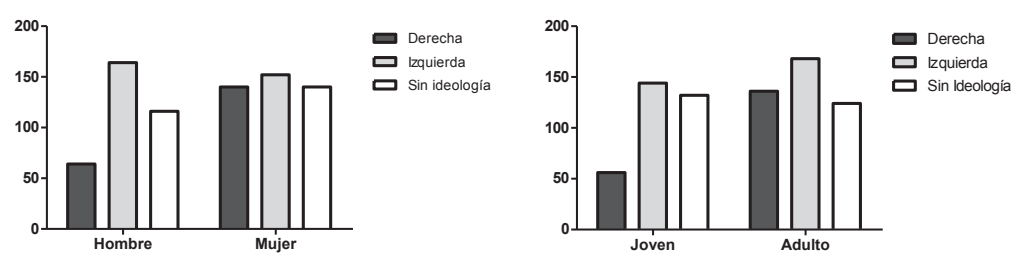

Figuras 3 y 8 . Frecuencias de participantes según ideología política y expectativas ambientales respecto de futuros gobiernos por sexo y edad
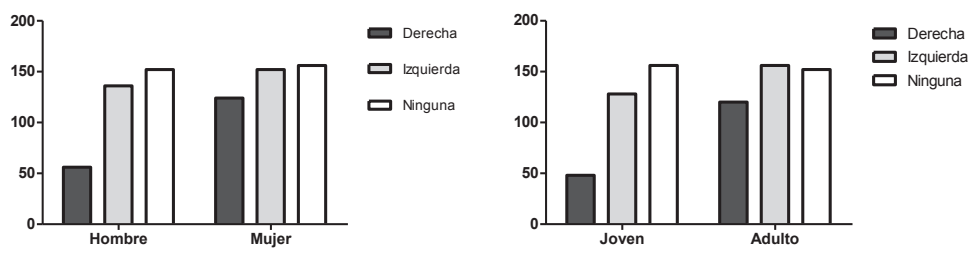

Figuras 4 y 9. Participantes pro o contra construcción de centrales nucleares en Chile por sexo y edad
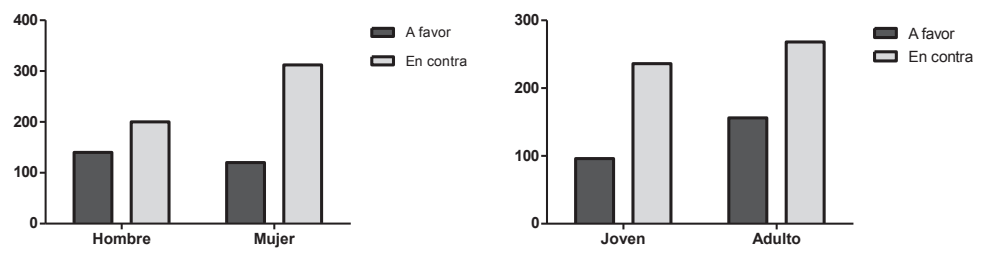

Figuras 5 y 10 . Auto-percepción pensamiento ambiental en Chile por sexo y edad
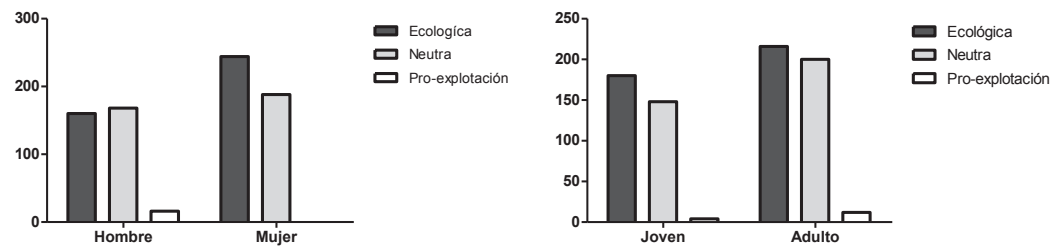